\title{
Fluorescence Activated Cell Sorting
}

National Cancer Institute

\section{Source}

National Cancer Institute. Fluorescence Activated Cell Sorting. NCI Thesaurus. Code C17353.

Selection and deposition of individual cells of a particular phenotype from a mixed population into a separate tube or tissue culture plate by the use of a fluorescenceactivated cell sorter (FACS) and fluorescently-labeled antibodies specific for surface molecules on the cells to be sorted. 